\title{
Multiple hepatocellular carcinomas: liver resection or transcatheter arterial chemoembolization?
}

\author{
Yin Lei ${ }^{1}$, Lau Wan Yee ${ }^{2}$, Wei-Ping Zhou ${ }^{1}$ \\ ${ }^{1}$ The Third Department of Hepatic Surgery, Eastern Hepatobiliary Surgery Hospital, Second Military Medical University, Shanghai 200438, China; \\ ${ }^{2}$ Faculty of Medicine, The Chinese University of Hong Kong, Prince of Wales Hospital, Shatin, Hong Kong, China \\ Correspondence to: Wei-Ping Zhou. The Third Department of Hepatic Surgery, Eastern Hepatobiliary Surgery Hospital, Second Military Medical \\ University, Shanghai 200438, China. Email: EHPHWP@126.com. \\ Comment on: Fukami Y, Kaneoka Y, Maeda A, et al. Liver Resection for Multiple Hepatocellular Carcinomas: A Japanese Nationwide Survey. Ann \\ Surg 2019. [Epub ahead of print].
}

Submitted Apr 24, 2019. Accepted for publication May 08, 2019.

doi: 10.21037/hbsn.2019.05.13

View this article at: http://dx.doi.org/10.21037/hbsn.2019.05.13

The article by Fukami et al. published in the Annals of Surgery is a detailed study using a very large sample size to discuss on the treatment strategies for multiple hepatocellular carcinoma (HCC) (1). Although this is a retrospective study, the authors used propensity score matching and other methods to draw interesting conclusions.

The American Association for the Study of Liver Disease (AASLD) and European Association for Study of the Liver (EASL) guidelines recommend liver transplantation (LT) to provide the best survival benefit for multiple HCC within the Milan criteria, and transcatheter arterial chemoembolization (TACE) to be the best choice for multiple HCC outside of the Milan criteria $(2,3)$.

Since the Barcelona Clinic Liver Cancer (BCLC) system was established 20 years ago (4), management of HCC has become more standardized. The publication of a large number of studies on HCC every year helps also to promote and improve clinical practice guidelines for HCC. The EASL released its HCC guidelines in 2001, 2012 and 2018, and announced that an updated version will be released in 2023.Although the BCLC guidelines, which is the core of the guidelines, have been developed for nearly 20 years, they have not changed much.

In the latest BCLC guidelines of HCC (2), a proportion of multiple HCC (two or three nodules, each $<3 \mathrm{~cm}$ in diameter) is defined as BCLC stage A, and the recommended treatment is LT or tumor ablation. With insufficient available data, the natural outcome of these patients was estimated to have a median survival of 36 months (5). In this recent article by Fukami et al. published in the Annals of Surgery, the authors divided multiple HCCs of up to 3 tumors according to tumor size into the $<30 \mathrm{~mm}$ group and the $\geq 30 \mathrm{~mm}$ group. Liver resection (LR) yielded better survival outcomes than TACE in both the two groups. My major criticism on this article is that the authors should compare the survival outcomes of LR with LT or with ablation for the $<30 \mathrm{~mm}$ group, rather than comparing with TACE, as LT and ablation are the recommended treatments for multiple HCC in the BCLC stage A. The authors should give an explanation as to why they compared LT with TACE.

Although the BCLC staging system is now widely used, there are still some controversial issues, especially on the treatment of HCC in the BCLC B/C stage. Some recently published studies have focused on these controversies. In the article by Fukami et al., the multiple HCC with up to 3 tumors and $\geq 30 \mathrm{~mm}$ group should be classified as BCLC stage $\mathrm{B}$ with the recommended treatment by the BCLC guidelines being TACE. However, this recommendation was based on 2 RCTs and 1 systematic review which suggested TACE could provide survival benefit when compared with palliative treatment. There have been insufficient data to compare TACE with other treatments, especially with LR. Recently many studies have emerged to suggest LR to provide better long-term survival outcomes than TACE for patients with BCLC stage B HCC (6-8).

The controversies on BCLC stage B HCC have led 
Bolondi et al. (9) to propose to divide BCLC stage B into the following subgroups: B1, B2, B3 and B4. As B3/ B4 was defined to have a Child score of $\geq 7$, the multiple HCC in the study by Fukami et al. should be classified in the $\mathrm{B} 1$ or $\mathrm{B} 2$ subgroups. Both the study by Bolondi et al. and another clinical study focusing on the BCLC stage B subgroups (10) reached a similar conclusion that the overall survival of patients with subgroup B1/B2 after LR was better than TACE. In fact, many centers which performed LR on stage B HCC achieved good long-term survival results. However, no high evidence-based level clinical studies have been conducted until the study reported by Yin et al. (6). This was the first well-designed RCT on stage B HCC. The results showed LR to provide better long-term survival outcomes when compared with TACE. Using the subgroup staging of Bolondi et al., the patients in the study by Yin et al. had stage B1/B2. Both the studies by Fukami et al. and Yin et al. led to the same conclusion: LR resulted in better long-term survival outcomes than TACE for patients with multiple HCCs with subgroup B1/B2 HCC staging.

Although Fukami et al. claimed LR provided the best survival outcomes for patients with multiple HCCs either within or beyond the Milan criteria, the data were derived from 4 retrospective clinical studies, 3 of which did not separately classify stage B HCC from the other stages. Although the authors claimed this to be the largest case series of multiple HCC from a nationwide survey, the study has the inherent defect of a retrospective study. Japan's strict requirements on LT for HCC, and the differences in surgical levels and clinical treatment strategies among institutions could lead to biases despite the use of propensity score matching analysis. In the future, more high-quality evidence-based medical evidences on stage B HCC are needed to determine the role of LR in the treatment of stage B HCC.

Although many published clinical studies have supported the important role of LR in the treatment of multiple HCC, and some studies even considered LR as the better choice for stage B HCC than TACE, there are still many aspects that need to be further studied. First, the range of stage $B$ HCC is wide and stage B HCC should further be divided into its subtypes according to tumor size, tumor number, and Child score. The significance and value of the many subgroups of stage B HCC should be verified. Secondly, using the different subgroups of stage $B$, prospective studies comparing the different treatment regimens using LR,
TACE, RFA, LT, chemotherapy, or sorafenib are needed. Finally, based on the results of these studies, the treatment guidelines for stage B HCC should further be refined. As Roayaie (11) once said "guidelines are not carved in stone and are meant to be modified as new data comes to light".

\section{Acknowledgments}

Funding: This study was funded by the National Natural Science Foundation for Young Scientists of China (No. 81602466) and Foundation of Shanghai Health and Family Planning Commission (No. 201640164).

\section{Footnote}

Conflicts of Interest: The authors have no conflicts of interest to declare.

\section{References}

1. Fukami $Y$, Kaneoka Y, Maeda A, et al. Liver Resection for Multiple Hepatocellular Carcinomas: A Japanese Nationwide Survey. Ann Surg 2019. [Epub ahead of print].

2. European Association for the Study of the Liver. EASL Clinical Practice Guidelines: Management of hepatocellular carcinoma. J Hepatol 2018;69:182-236.

3. Heimbach JK, Kulik LM, Finn RS, et al. AASLD guidelines for the treatment of hepatocellular carcinoma. Hepatology 2018;67:358-80.

4. Llovet JM, Brú C, Bruix J. Prognosis of hepatocellular carcinoma: the BCLC staging classification. Semin Liver Dis 1999;19:329-38.

5. Forner A, Reig ME, de Lope CR, et al. Current strategy for staging and treatment: the BCLC update and future prospects. Semin Liver Dis 2010;30:61-74.

6. Yin L, Li H, Li AJ, et al. Partial hepatectomy vs. transcatheter arterial chemoembolization for resectable multiple hepatocellular carcinoma beyond Milan Criteria: a RCT. J Hepatol 2014;61:82-8.

7. Ho MC, Huang GT, Tsang YM, et al. Liver resection improves the survival of patients with multiple hepatocellular carcinomas. Ann Surg Oncol 2009;16:848-55.

8. Kim H, Ahn SW, Hong SK, et al. Survival benefit of liver resection for Barcelona Clinic Liver Cancer stage B hepatocellular carcinoma. Br J Surg 2017;104:1045-52.

9. Bolondi L, Burroughs A, Dufour JF, et al. Heterogeneity 
of patients with intermediate (BCLC B) Hepatocellular Carcinoma: proposal for a subclassification to facilitate treatment decisions. Semin Liver Dis 2012;32:348-59.

10. Xu QG, Yuan SX, Tao QF, et al. A novel HBx genotype serves as a preoperative predictor and fails to activate

Cite this article as: Lei Y, Yee LW, Zhou WP. Multiple hepatocellular carcinomas: liver resection or transcatheter arterial chemoembolization? HepatoBiliary Surg Nutr 2019;8(5):519-521. doi: 10.21037/hbsn.2019.05.13 the JAK1/STATs pathway in hepatocellular carcinoma. J Hepatol 2019;70:904-17.

11. Roayaie S. TACE vs. surgical resection for BCLC stage B HCC. J Hepatol 2014;61:3-4. 